\title{
Treatment of a Total Obstructive Anastomosis Stricture Using a Transanal Laparoscopic Approach and Intraoperative Colonoscopic Balloon Dilatation
}

\author{
Jae Young Kwak ${ }^{1}$, Kwan Mo Yang ${ }^{1}$, Hyun Il Seo ${ }^{2}$ \\ Departments of ${ }^{1}$ Surgery and ${ }^{2}$ Internal Medicine, Gangneung Asan Hospital, University of Ulsan College of Medicine, Gangneung, Korea
}

An anastomosis stricture with a total obstruction is rare and treatment options are variable. We describe our experience with a combination of a single port transanal laparoscopic approach and intraoperative colonoscopic balloon dilatation. The patient was a 48-year-old man with rectal cancer. A laparoscopic single port lower anterior resection and diverting ileostomy were performed followed by a colon study and ileostomy takedown. The colon study and sigmoidoscopy revealed total obstruction of the rectum at the anastomosis level. We employed a transanal approach using a single port to correct this. We located the anastomosis stricture site and generated a lumen using a dissector and electocautery method to insert the balloon device. Colonoscopic balloon dilatation was subsequently successful. The patient was discharged with no postoperative complications. A laparoscopic single port transanal approach with an intraoperative colonoscopic balloon dilatation is a viable alternative approach to treating an anastomosis stricture of the rectum.

Keywords: Anastomosis; Stricture; Transanal; Balloon

\section{INTRODUCTION}

A rectal anastomosis stricture is a rare but not insignificant possible complication of rectal surgery. Its incidence varies from $2 \%$ to $30 \%[1-3]$ and is higher in patients undergoing postoperative radiotherapy and stapled rather than hand-sewn coloanal anastomosis [1]. The management of a rectal anastomosis stricture varies from major pelvic surgery to local revision. Garcea et al. [4] reviewed these approaches and reported that the most common intervention was dilation only, with other treatments including pelvic revision surgery, fecal diversion, transanal microsurgery, strictureplasty, Hegar dilatation, electrocauterization, and mechanical

Received: Nov 5, 2019 • Revised: Feb 11, 2020 • Accepted: Feb 27, 2020 Correspondence to: Hyun II Seo, M.D.

Department of Internal Medicine, Gangneung Asan Hospital, University of Ulsan College of Medicine, 38 Bangdong-gil, Sacheon-myeon, Gangneung 25440, Korea

Tel: +82-33-610-3945, Fax: +82-33-644-5495

E-mail: reshi@hanmail.net

ORCID: https://orcid.org/0000-0002-0339-5031

(C) 2020 The Korean Society of Coloproctology

This is an open-access article distributed under the terms of the Creative Commons Attribution NonCommercial License (https://creativecommons.org/licenses/by-nc/4.0) which permits unrestricted noncommercial use, distribution, and reproduction in any medium, provided the original work is properly cited. stenting. Anastomosis strictures also have several different types [5] which need to be considered when selecting the most appropriate treatment option.

Our present case report describes our experience with a combination treatment of a single port laparoscopic transanal approach and intraoperative colonoscopic balloon dilatation in a patient with an anastomosis site stricture with near-total obstruction.

\section{CASE REPORT}

A 48-year-old man was referred to our hospital for the evaluation of hematochezia for 10 days. A colonoscopy revealed a polypoid rectal adenocarcinoma at $10 \mathrm{~cm}$ above the anal verge. A computed tomography and magnetic resonance imaging revealed no evidence of pericolic fat infiltration, lymph node metastasis, or distant metastasis. The patient wanted surgical treatment than endoscopic resection. He underwent a single port laparoscopic low anterior resection and diverting ileostomy. Inferior mesenteric artery was ligated at the level of origin and rectal anastomosis was performed using a double-stapling technique with $28-\mathrm{mm}$ EEA Circular Stapler (Medtronic Inc., Minneapolis, MN, USA) at the level of $8 \mathrm{~cm}$ above anal verge. He was hemodynamically stable after surgery with no detectable postoperative complications. 


\section{Annals of Intraoperative Colonoscopic Balloon Dilatation \\ Coloproctology Jae Young Kwak, et al.}

The tumor pathology indicated a TisN0M0 lesion. The patient made a full recovery and was discharged 7 days later. At 2 months postsurgery, a colon study was performed for an ileostomy takedown but revealed a total obstruction of the rectum at the anastomosis level. We could exclude other reasons for anastomosis obstruction such as tumor or other extraluminal problem with abdominopelvic computed tomography scan. A subsequent sigmoidoscopy confirmed that an anastomosis site stricture was the cause (Fig. 1).

We decided to employ a transanal approach using a single port (GelPOINT Path, Applied Medical, Rancho Santa Margarita, CA, USA) to correct this obstruction. We located the anastomosis stricture site and generated a lumen using a dissector and electrocautery method to insert a balloon device (Fig. 2). Through-the- scope balloon dilatation was then done under endoscopic visualization of the stenosis. A controlled radial expansion balloon dilator (CRE PRO Wireguided, 12- $\mathrm{mm} / 13.5-\mathrm{mm} / 15-\mathrm{mm}$ diameter; Boston Scientific, Cork, Ireland) was inserted through the stricture and distended for 1 minute for each diameter. No fluoroscopic control was used during the balloon dilation and the procedure was completed successfully with no complications. The patient was discharged 7 days after this operation without postoperative complications. A follow-up 3 months later found no other adverse events with normal sigmoidoscopic findings and ileostomy takedown was done successfully (Fig. 3).

The Institutional Review Board of Gangneung Asan Hospital approved this study and waived the informed consent requirement (No. GNAH 2019-05-011).
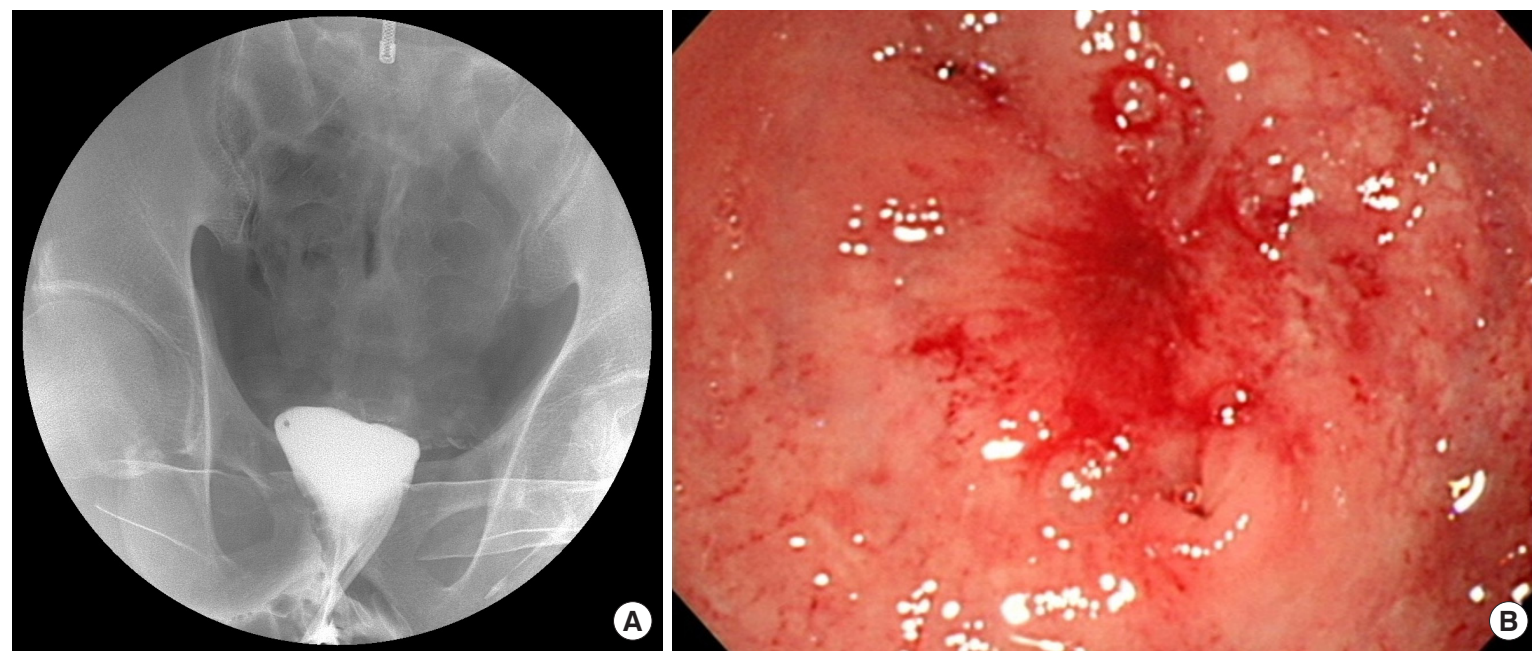

Fig. 1. Colon study (A) and sigmoidoscopy (B) of the patient revealing a total obstruction of the rectum at the anastomosis level.
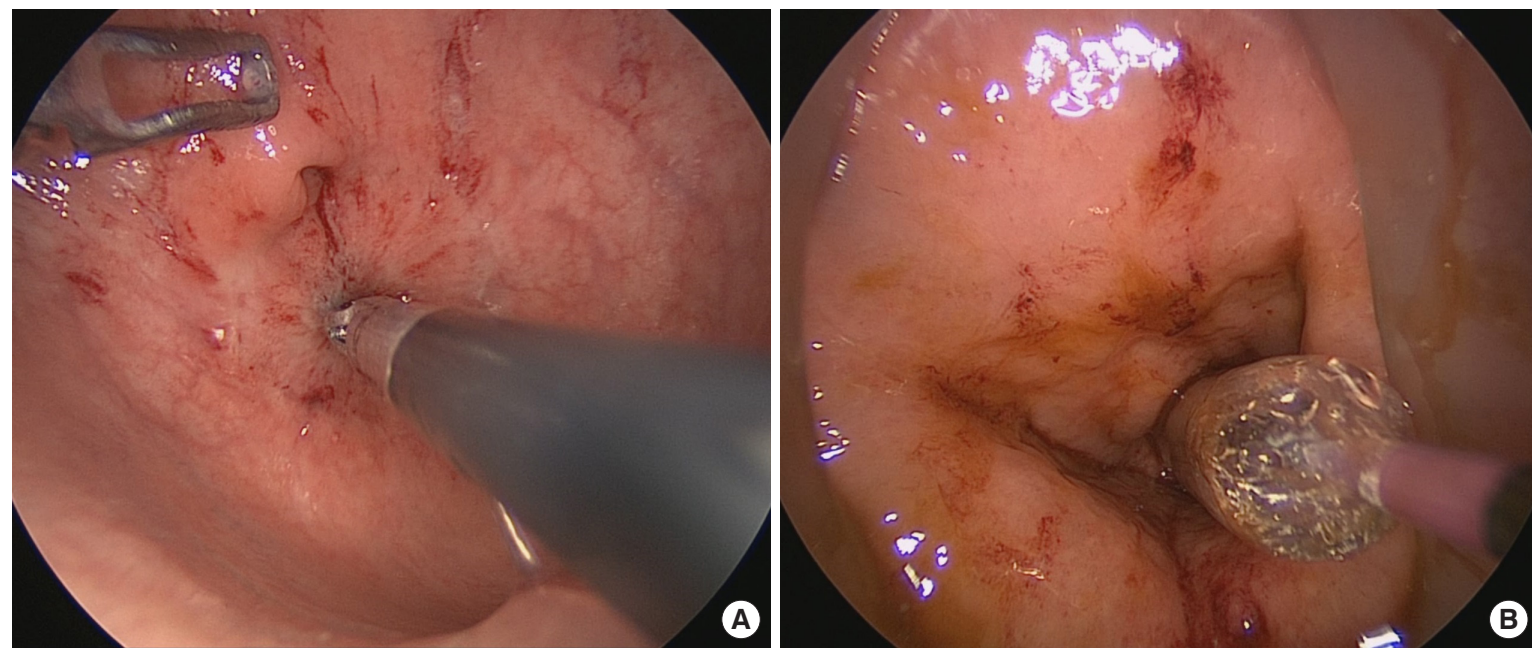

Fig. 2. Transanal electrocautery (A) was used to generate a lumen followed by colonoscopic balloon dilatation (B). 


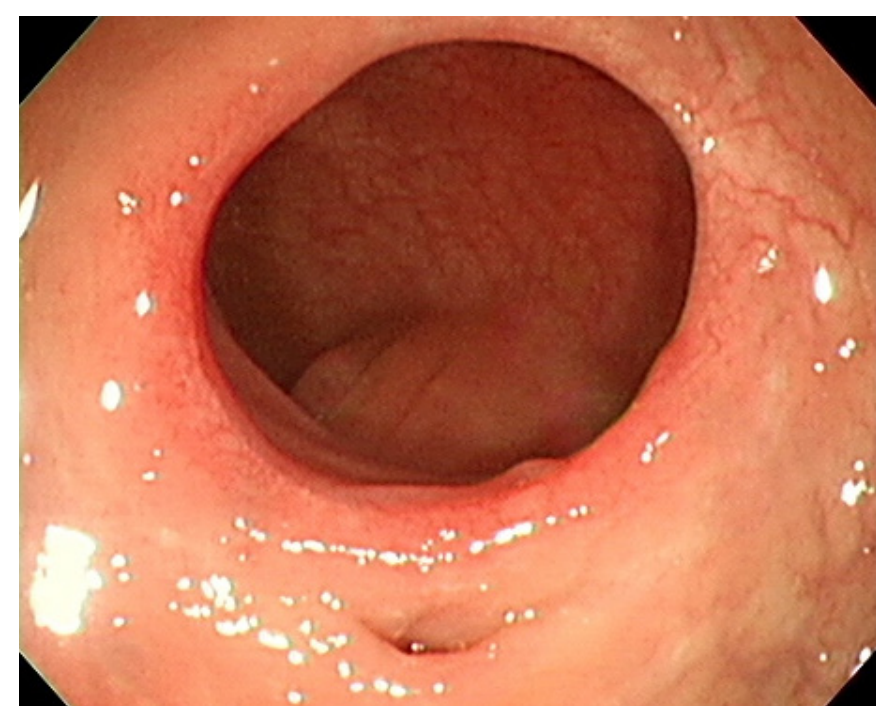

Fig. 3. Sigmoidoscopy findings at 3 months later after the procedure.

\section{DISCUSSION}

The term anastomosis stricture can be used to refer to a narrowing in the colon that prevents a normal passage of the stools, but there is no clear definition. Because of this lack of a consensus in defining a 'true' anastomosis stricture, the incidence of this complication has been reported at a wide range, from $2 \%$ to $30 \%$ [1-3]. Most reported instances of anastomosis stenosis have occurred within 6 months of surgery [6].

Ischemia of the suture line, leakage, inflammation, and hemorrhage have been proposed as underlying pathophysiological mechanisms of postoperative rectal anastomosis strictures, with risk factors reported to include stapled anastomosis, radiotherapy, diverticulitis, and the level and tension of the anastomosis [1-3, 6, 7]. A stapled anastomosis is associated with higher levels of collagen deposition and inflammation, which may lead to stenosis formation [8]. Radiotherapy can also cause histologic alterations such as obliterative endarteritis, tissue ischemia and necrosis, and submucosal collagen deposition, and these changes can lead to transmural fibrosis and stricture formation [9]. Diverticulitis has also been described as a major risk factor for stenosis. Polese et al. [2] reported a 5-fold higher risk of stenosis in patients with diverticulitis compared to those with neoplasia.

Various treatment options for an anastomosis stricture have been reported including balloon dilatation, pelvic revision surgery, transanal strictureplasty, electrocautery methods, and mechanical stenting [4]. Endoscopic balloon dilatation has been the most commonly reported technique with the described success rates ranging from $33 \%$ to $100 \%[4,10,11]$ and a reported repeat dilatation rate of up to $88 \%$, with a restricture rate of up to $30 \%$. Perforation and bleeding are the most serious complications of this procedure, with an occurrence rate of $5 \%$, with other possible adverse events including abscess, transient septicemia, and technical failure [4]. The transanal strictureplasty technique using a circular stapler has a reported success rate of $64 \%$. However, this particular method is not really feasible in cases of very tight stenosis, which a stapler cannot pass through. Moreover, there is a higher risk of fistula formation and ischemic change complications with this approach $[4,10]$. Stenting has been rarely reported as the chosen management option for an anastomosis stricture as migration, erosion, pressure necrosis, and bleeding are considered to be high-risk complications of this method [4]. Surgical management with laparotomy requires extensive pelvic dissection and is therefore a potentially dangerous procedure. It may be needed however in cases of dilatation or endoscopic technique failure, transanal approach failure, or for severe long segment stenosis. Resection of the anastomosis site and reconstruction with a new stapled anastomosis can be a surgical option in patients with favorable local conditions; if the conditions are unfavorable, reconstruction with a straight coloanal anastomosis or permanent colostomy are possible surgical options [3].

An anastomosis stricture with a total obstruction is a rare but serious complication for which a consensus management option is yet to be established. In our current case, it was difficult to preoperatively assess the length and severity of the stenosis. We therefore chose and successfully applied a combination treatment involving a transanal laparoscopy to create a lumen and prevent bleeding or perforation and balloon dilatation. We introduce carefully our experience with this method that it is a viable alternative for the treatment of anastomosis stricture with total obstruction of the rectum.

\section{CONFLICT OF INTEREST}

No potential conflict of interest relevant to this article was reported.

\section{REFERENCES}

1. Neutzling CB, Lustosa SA, Proenca IM, da Silva EM, Matos D. Stapled versus handsewn methods for colorectal anastomosis surgery. Cochrane Database Syst Rev 2012;(2):CD003144.

2. Polese L, Vecchiato M, Frigo AC, Sarzo G, Cadrobbi R, Rizzato R, et al. Risk factors for colorectal anastomotic stenoses and their impact on quality of life: what are the lessons to learn? Colorectal Dis 2012;14:e124-8.

3. Schlegel RD, Dehni N, Parc R, Caplin S, Tiret E. Results of reoperations in colorectal anastomotic strictures. Dis Colon Rectum 2001;44:1464-8.

4. Garcea G, Sutton CD, Lloyd TD, Jameson J, Scott A, Kelly MJ. Management of benign rectal strictures: a review of present therapeutic procedures. Dis Colon Rectum 2003;46:1451-60.

5. Milsom JW, Mazier WP. Classification and management of postsurgical anal stenosis. Surg Gynecol Obstet 1986;163:60-4. 


\section{Coloproctology Jae Young Kwak, et al.}

6. Sartori A, De Luca M, Fiscon V, Frego M; CANSAS study working group, Portale G. Retrospective multicenter study of post-operative stenosis after stapled colorectal anastomosis. Updates Surg 2019;71:539-42.

7. Sauer R, Becker H, Hohenberger W, Rödel C, Wittekind C, Fietkau R, et al. Preoperative versus postoperative chemoradiotherapy for rectal cancer. N Engl J Med 2004;351:1731-40.

8. Dziki AJ, Duncan MD, Harmon JW, Saini N, Malthaner RA, Trad $\mathrm{KS}$, et al. Advantages of handsewn over stapled bowel anastomosis. Dis Colon Rectum 1991;34:442-8.
9. Theis VS, Sripadam R, Ramani V, Lal S. Chronic radiation enteritis. Clin Oncol (R Coll Radiol) 2010;22:70-83.

10. Kraenzler A, Maggiori L, Pittet O, Alyami MS, Prost À la Denise J, Panis Y. Anastomotic stenosis after coloanal, colorectal and ileoanal anastomosis: what is the best management? Colorectal Dis 2017;19:O90-6.

11. Ragg J, Garimella V, Cast J, Hunter IA, Hartley JE. Balloon dilatation of benign rectal anastomotic strictures: a review. Dig Surg 2012;29:287-91. 